\title{
Potential Role of CC Chemokines and their Receptors in the Development of Respiratory Diseases
}

\author{
Lin Tong, YaoLi Wang, Jing Bi, Dong Yang, ChunXue Bai* and XiangDong Wang*
}

Department of Pulmonary Medicine, Zhongshan Hospital, Fudan University, Shanghai, China

\begin{abstract}
Increasing evidence suggests that the chemokine system coordinates leukocyte migration in immunity and inflammation, involving in the pathogenesis of many pulmonary diseases. Chemokines are small proteins which interact with specific receptors to exert their chemotactic functions for inflammatory cells and constitutive cells. Among the complex system, CC chemokine receptors (CCRs) represent a subfamily of chemokine receptor, which is considered to play an important role in the pathogenesis of diseases characterized by disordered inflammation and immunity, among which some respiratory diseases, such as asthma, chronic obstructive pulmonary disease, acute lung injury, comprise one important part. The present paper reviewed the research of possible relationship and progress between this system and human lung diseases. Chemokines and their receptors are essential components of Th1- and Th2-mediated responses via the recruitment of lymphocytes and macrophages. The local expression of homeostatic chemokines in pulmonary also plays an important role in protective immune responses. Thus, it is important to understand the potential role of CCRs in respiratory diseases and it may provide exciting new targets for therapeutic intervention.
\end{abstract}

Keywords: Chemokines, CCR, lung, asthma, COPD.

\section{INTRODUCTION}

Chemokine receptors are members of G-protein-coupled receptor superfamilies, which are characterized by seventransmembrane-spanning protein coupled to heterotrimeric $\mathrm{G}$ protein. As their ligands, chemokines are a large family of chemoattractic cytokines, playing an important role during episodes of tissue inflammation and injury, by binding to specific chemokine receptors to activate and recruit a wide variety of cell types, notably leukocytes. Both chemokines and their receptors have been implicated in a host of clinically important diseases, including a series of respiratory diseases. The current review focuses more on understanding the role of CCRs in the pathogenesis of respiratory diseases (Table 1) and possible interventional therapeutic strategies being used for patients.

Chemokines are small proteins with four conserved cysteines forming two essential disulphide bonds (Cys1-Cys3 and Cys2-Cys4). The approximately 50 chemokines and 20 receptors identified to date are classified into four families, on the basis of the pattern of the first two of four cysteine residues of the ligand. The large $\mathrm{CC}$ chemokine family consists of chemokines with the first two cysteine residues adjacent to each other $[1,2]$. The CCR family comprises 10 receptors (CCR1-10) with their 25 ligands (CCL), dominating about half of the chemokine and chemokine receptor family. Binding to chemokine receptors results in the dissociation of $\mathrm{G}_{\alpha \mathrm{I}}$ and $\mathrm{G}_{\beta \gamma}$ subunits of the heterotrimeric $\mathrm{G}$ proteins (Fig. 1). This leads to calcium flux and activation of the phosphatidylinositol 3-kinase and the small Rho GTPases signaling

*Address correspondence to these authors at the Department of Pulmonary Medicine, Zhongshan Hospital, Fudan University, Shanghai, 200032, China; Tel: +86 216404 1990, ext. 2445; E-mail: xiangdong.wang@telia.com; bai.chunxue@zs-hospital.sh.cn pathways and regulates gradient sensing and F-actin polymerization at the leading edge of migrating cell, responsible for cell contraction, adhesion and activation [1]. Their main function is considered to mediate and direct the trafficking and migration of monocytes and lymphocytes. Chemokine receptors are not only expressed by circulating cells but also by tissue resident cells, including epithelia, endothelia, stromal cells, neurons and smooth muscle, and their expression is upregulated in various inflammatory conditions [3].

The lung is a unique organ supplied with blood from both pulmonary and systemic circulations, that deliver blood to the parenchyma and the airways, respectively. Due to the low pressure of the pulmonary system, leukocyte rolling and firm adherence are not required for extravasation. It is suggested that a weak chemoattractic signal per se can induce and mediate migration into the underlying tissue [4]. A number of respiratory diseases, such as asthma, chronic obstructive pulmonary disease (COPD), acute lung injury (ALI) and acute respiratory distress syndrome (ARDS), share common pathophysiological processes, like inflammation, independent upon either acute or chronic. Unregulated inflammatory process may lead to histological alterations like fibrosis and remodeling and impairment of lung function. The relationship between chemokine system and respiratory diseases as the new scope for further research and therapeutic targets remains unclear.

\section{ROLE IN ASTHMA}

Asthma is a complex immunological and inflammatory disease characterized by the presence of airway inflammation, tissue remodeling and bronchial hyperresponsiveness. It remains to understand the development and interaction of those three key features in the pathogenesis of the lung disease. The inflammatory response characteristically comprises activated T helper type 2 (Th2) lymphocytes, eosino- 
Table 1. Role of CC Chemokines and Related CCRs in the Pathogenesis of Respiratory Diseases

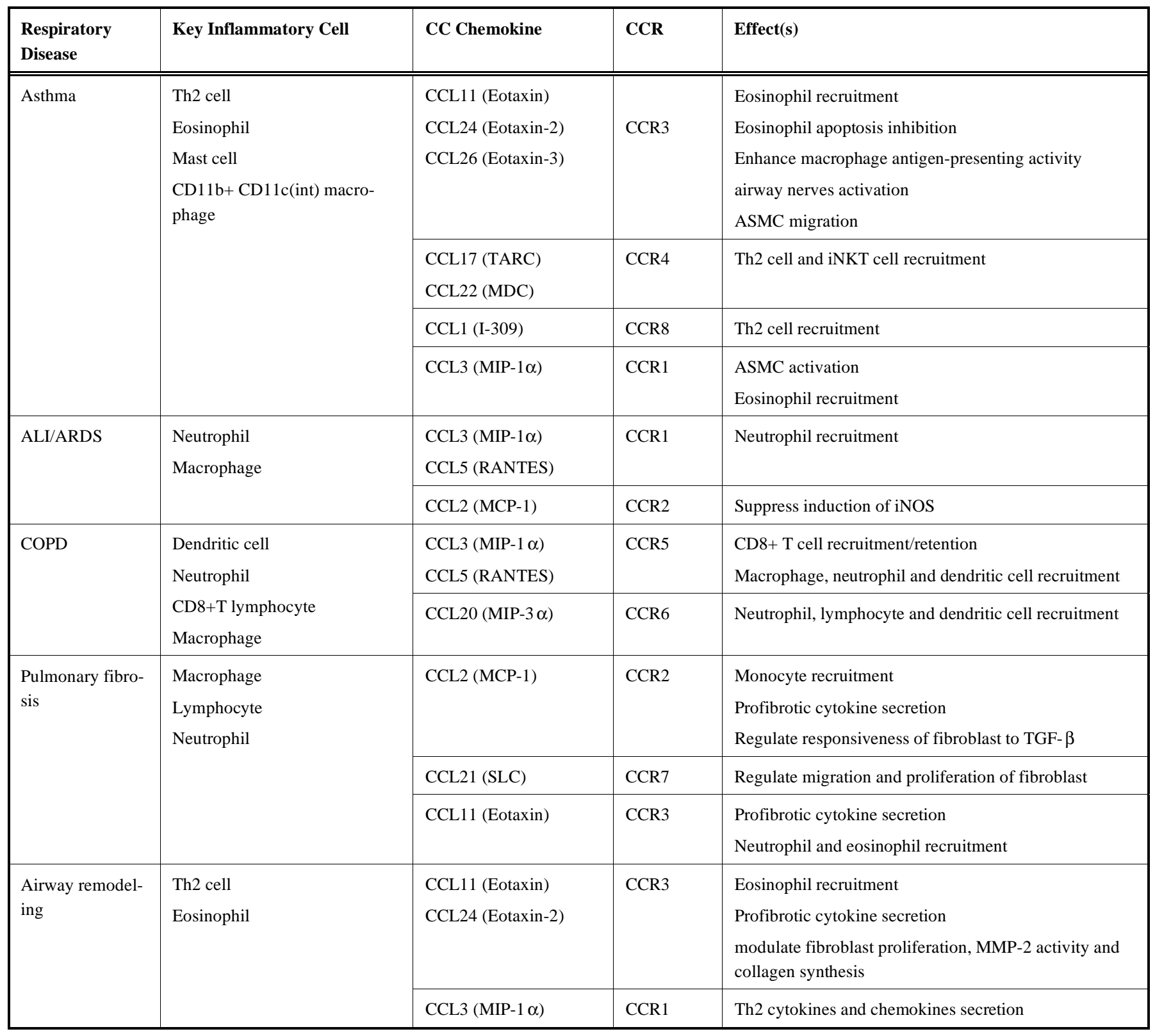

phils and activated mast cells, associated with the etiology [5]. Such specific accumulation of distinct leukocytes is mediated by a number of chemokines, as listed in Table 2. There is a strong evidence from both experimental and clinical studies to confirm the role of chemokines and their receptors, (Fig. 2) including CCR3, CCR4, CCR8, and CCR1, in the development of allergic inflammation [6,7].

\section{CCR3}

The CC chemokine receptor 3 (CCR3) is expressed on eosinophils, basophils, mast cells, Th2 cells, and interestingly on bronchial epithelial cells and smooth muscle cells. It has been suggested that CCR3 and its ligands may help to co-localize the major cellular components into the lungs during the allergic airway response [8,9]. CCR3/eotaxin (including CCL11, CCL24, CCL26) axis is essential in eosinophils recruitment to the asthmatic lungs. Increased expression of
CCL11 and CCL24 at both mRNA and protein levels has been observed at sites of allergic inflammation in both atopic and non-atopic asthmatics and also in the sputum, whilst increased CCL26 expression was noticed in the asthmatic lung 24 hours following allergen challenge [10]. Although there existed controversies about the role of CCR3 on the induction of lung eosinophilia [11,12], allergen-induced pulmonary eosinophilia has been proposed to be primarily mediated by CCR3 and its ligands eotaxin-1 and eotaxin-2. Eotaxin-2 has a dominant role in OVA-induced airway eosinophilia [13]. In addition to pro-inflammatory activity, eosinophils may interact with airway nerves through CCR3 signaling, increasing acetylcholine release and involving in bronchoconstriction [14].

Furthermore, CCR3/eotaxin axis may have additional functions other than eosinophils recruitment. Farahi et al. recently suggests that human pulmonary artery endothelial 


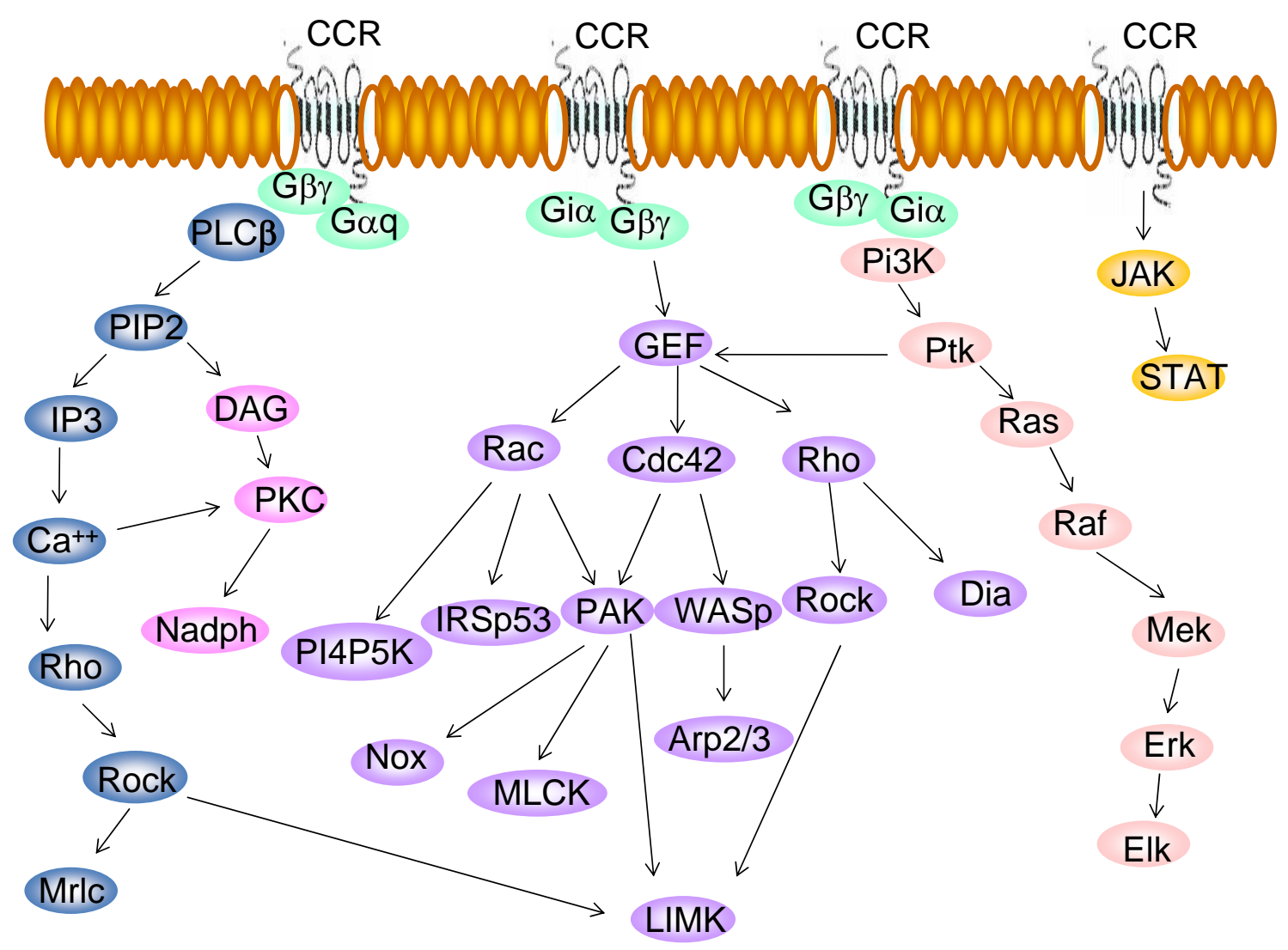

Fig. (1). Signaling pathways of CC chemokine receptor (CCR). PLC: phospholipase C; PIP2: phosphatidylinositol-4,5-bisphosphate; IP3: inositol 1,4,5-trisphosphate; Rho: ras homolog gene family; ROCK: Rho-associated coiled-coil-containing kinase; Mrlc: myosin regulatory light chain; DAG: diacylglycerol; PKC: protein kinase C; Nadph: nicotinamide adenine dinucleotide phosphate; LIMK: LIM-motifcontaining kinase; GEF: guanine nucleotide-exchange factor; Cdc42: small GTPase of the Rho family; Dia: Diaphanous formin protein family; MLCK: myosin light-chain kinase; PI4P5K: phosphoinositide 4-phosphate 5-kinase; WASp: Wiskott Aldrich Syndrome protein; PAK: p21-activated kinases; IRSp53: Insulin receptor substrate protein 53; Nox: NADPH oxidase; Pi3K: phosphoinositide 3-kinase; Ptk, protein tyrosine kinase; ERK: extracellular-signal-regulated kinase; MEK: mitogen-activated protein kinase/ERK kinase; JAK: Janus kinase; STAT: signal transduction and activator of transcription.

Table 2. Polymorphisms and Pulmonary Diseases in the CC Subfamily

\begin{tabular}{|c|c|c|c|c|}
\hline Ligand & Polymorphism & Location & Symbol & Disease Involved \\
\hline \hline CCL1 & SNP (A/T) & Intron 2 & rs2282691 & Chronic obstructive pulmonary disease \\
\hline CCL2 & SNP -2518 (G/A) & Promoter & rs1024611 & Asthma, Pulmonary tuberculosis \\
\hline CCL5 & SNP -403 (G/A) & Promoter & rs2107538 & Allergic rhinitis, Atopy and Asthma \\
\hline CCL11 & SNP -576 (C/T) & Promoter & rs4795896 & Asthma \\
\hline CCL24 & SNP +179 (T/C) & Intron 1 & rs22402004 & Asthma \\
\hline CCL26 & SNP +77 (C/T) & Intron 2 & & \\
\hline
\end{tabular}

cells have a capacity of elaborating and secreting CCL11, inhibiting apoptosis in human eosinophils [15]. This indicates a mechanism for the aberrant survival of eosinophils in airway allergic inflammation. Additionally, CD11b(+) CD11c(int) macrophages expressing CCR3 as key proinflammatory cells were found to be both necessary and sufficient for allergen-specific $\mathrm{T}$ cell stimulation during devel- opment of eosinophia-dominated airway inflammation [16]. The expression of CCR3 on airway smooth muscle cells (ASMC) was increased in asthmatics, and that a CCR3 ligand such as eotaxin could induce migration of ASMC in vitro. Those results suggest that eotaxin could be involved in the increased smooth muscle mass observed in asthmatics through the activation of CCR3 [9]. ASMC has been consid- 


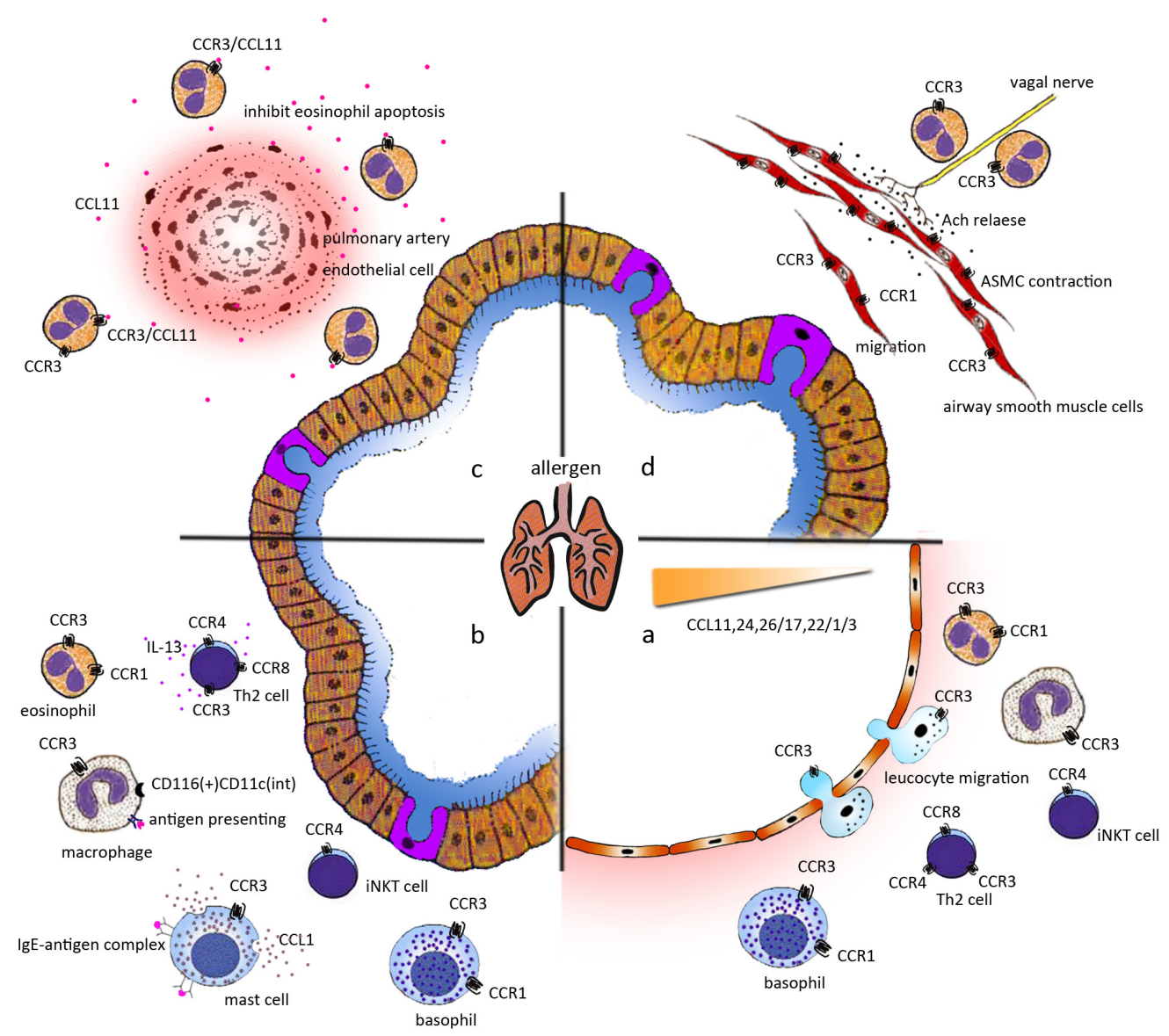

Fig. (2). Role of CCRs in the pathogenesis of asthma. Migration of distinct subsets of leukocyte is mediated by the chemokines gradient produced by allergen-challenged lung tissue (a). The accumulation of specific inflammatory cells occurs in the airway, including activated Th2 lymphocytes, eosinophils, activated mast cells, CD11b(+) CD11c(int) macrophages, iNKT cells, and basophils (b). Pulmonary vascular endothelial cells have a capacity to elaborate and secrete CCL11, to operate as a survival agent in human eosinophils (c). Additionally, the expression of CCR3 by airway smooth muscle cell (ASMC) is increased and CCR3 ligand induces the migration of ASMC. Eosinophils may interact with airway nerves through CCR3 signaling-mediated recruitment, increasing acetylcholine (Ach) release and bronchoconstriction (d).

ered to serve as a source of chemokines in allergen-induced airway inflammation that ultimately recruits and retains inflammatory cells and may amplify the process of inflammation [17].

On the basis of CCR3 contributions in the pathogenesis of asthma, CCR3 has attracted additional attentions as potential target for treating airway inflammation in asthma. Various strategies, including monoclonal antibody [18], small molecule CCR3 antagnists [19, 20], topical inhibition of CCR3 mRNA expression [21], have been proved to be effective in animal models, while efficacy and safety on human remains to be tested.

\section{CCR4}

CCR4 is the sole receptor identified for the chemokines CCL22/Monocyte Derived Chemokine and CCL17/Thymusand Activation-Regulated Chemokine. CCR4, together with
CCR3 and CCR8, characterizes polarized Th2 lymphocytes, which migrate selectively in response to its ligands produced by monocytes, dendritic cells, and airway epithelial cells. The production of CCR4 was elevated after the stimulation with Th2 cytokines, suggesting an amplification circuit of polarized Th2 responses and a role for CCR4 in allergic airway inflammation $[8,10,22,23]$. An absolute requirement of CCR4 for T-lymphocytes was noted during the induction and maintenance of airway inflammation in asthmatics [10]. The majority of T-lymphocytes present in bronchial biopsies from atopic asthmatics were CCR4-positive and were presumably recruited via CCL22 and CCL17 [22].

The pulmonary localization of iNKT cells critical for the induction of airway hyperreactivity required CCR4 expression on iNKT cells [24]. Experimental studies suggest that a more complex level of organization, with CCR4 marking a major subset of circulating non-intestinal memory $\mathrm{T}$ - 
lymphocytes of both Th1 and Th2 potential, acted as a major trafficking receptor for systemic memory T-lymphocytes [25]. The CCR4/CCL17/CCL22 axes have been reported to play a pivotal role in the late phase allergic reaction. Studies have shown that repeated allergen challenge results in an increase in the number of CCR4+ T-lymphocytes within the airways which can be reduced by treatment with specific blocking antibodies [10]. Another study on transgenic mice and antibody blockade revealed that CCL17-CCR4 interaction dramatically impaired the pulmonary antifungal response during fungal asthma in neutropenic mice [26].

\section{CCR8}

CCR8 is a specific receptor for the chemokine CCL1/I309 , which is the predominant chemokine secreted from IgEactivated human and mouse mast cells and was elevated in asthmatic airways. CCR8 is expressed by approximately $70 \%$ of CD4-positive $\mathrm{T}$ lymphocytes recruited to the asthmatic airways, and the number of CCR8-expressing cells increased 3-fold in the airways of asthmatics, as compared with normal volunteers [27], similar to the findings in earlier studies [22]. There was a conflicting result, suggesting that the CCR8-CCL1 axis was not important for Th2 cell recruitment to the inflamed lung [10].

A recent study showed that neutralization of CCL1 or CCR8 deficiency could reduce mucosal lung inflammation, airway hyperresponsiveness, and mucus hypersecretion to a similar degree as detected in mast cell-deficient mice. Adenoviral delivery of CCL1 to the lungs of mast cell-deficient mice restored airway hyperresponsiveness, lung inflammation, and mucus hypersecretion to the degree observed in wild-type mice. The consequences of CCR8 deficiency, including a marked reduction in Th2 cytokine levels, were comparable with those observed by depletion of CD4positive T lymphocytes [27]. Thus, mast cell-derived CCL1 and CCR8-expressing CD4(+) effector T lymphocytes play an essential role in orchestrating lung mucosal inflammatory responses. The enhanced innate immune response in the absence of CCR8 promoted the rapid clearance of fungal material from the lung, facilitating the remission of fungal asthma [28], suggesting that CCR8 might be an attractive target in fungal-allergic asthma and other fungal-associated pulmonary diseases.

\section{Other CCRs}

In addition, other CCRs have also been reported to be involved in the pathogenesis of allergic airway inflammation, such as CCR1, CCR2 [8, 29], CCR7 [30-33], CCR9 [34]. CCR1 is expressed on basophils, monocytes and memory $\mathrm{T}$ cells and has been reported to be expressed at high levels on the eosinophils of around $15-20 \%$ of asthmatics [10]. Presently, CCR1 is believed to be more involved in

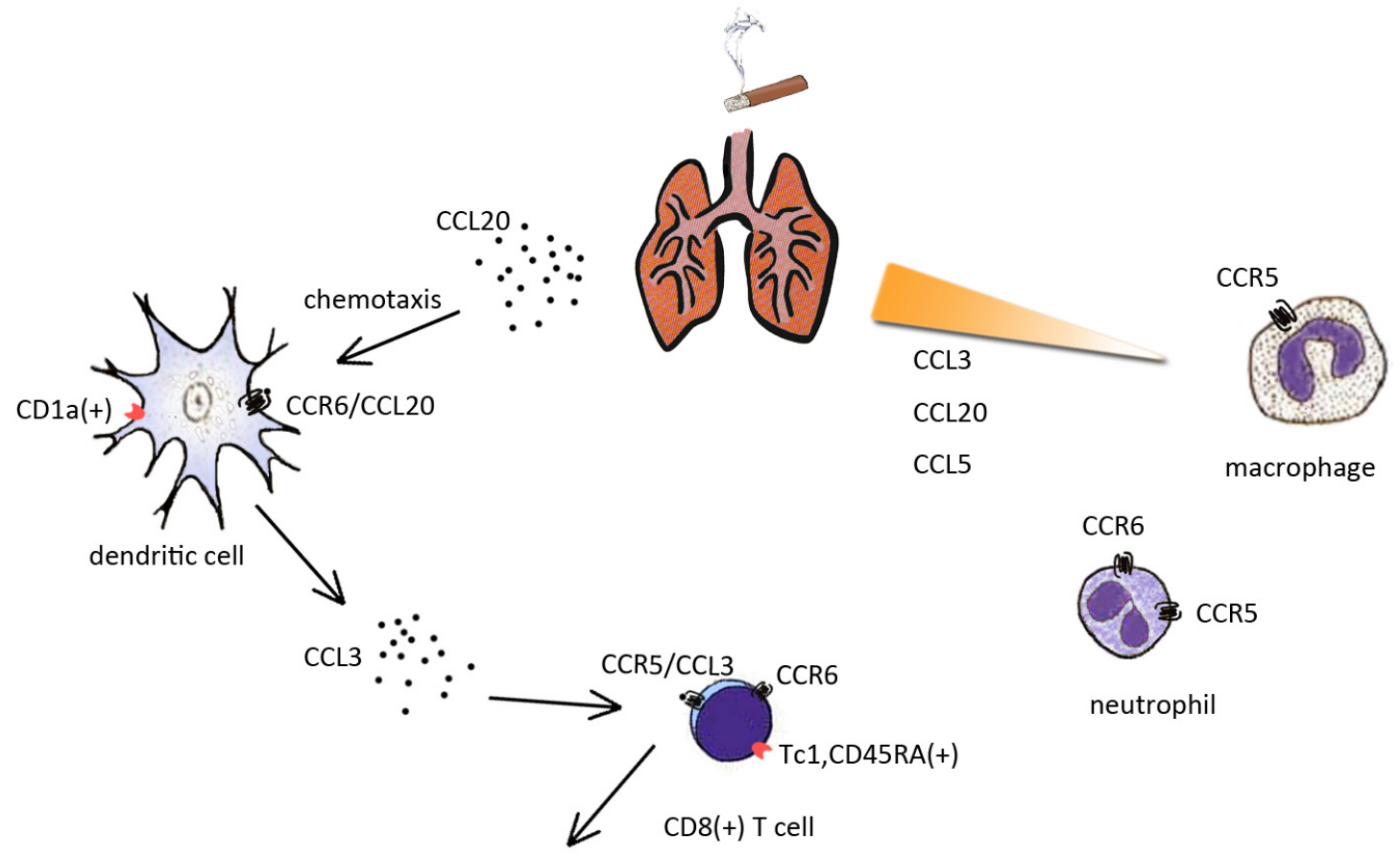

sustain CD8(+) T cell recruitment/retention

Fig. (3). Role of CCRs in the pathogenesis of COPD. CCL3(MIP-1 $\alpha$ ), CCL20(MIP-3 $\alpha$ ), CCL5(RANTES) are up-regulated in patients with COPD to attract specific inflammatory cells, like macrophages, dendritic cells (DCs), neutrophils and CD8(+) T-lymphocytes into the airway. Interaction between CCL20 and CCR6 provides a possible mechanism for accumulation of DCs in the lungs in COPD. CCL3 produced by $\mathrm{CD} 1 \mathrm{a}(+) \mathrm{DCs}$ potentially mediate CD8(+) T-cell infiltration during COPD progression, and CD1a(+) mucosal-associated DCs may sustain CD8(+) T-cell recruitment/retention. 
virally exacerbated allergic asthma than other types of asthma [35,36]. The expression of CCR1 and its functionality on ASMCs indicates that CCR1 may be involved in the pathogenesis of asthma, through the activation of ASMC by its ligands [37]. Therapeutic application was implied by the significant effect of nonpeptide CCR1 antagonist on fungusassociated allergic asthma [38]. CCR5 and CXCR3 have been reported to play important roles in the lung T-cell homing pathway, and may be potential targets for asthma therapy.

Several promising drugs, e.g. antisense oligonucleotide therapy targeting the receptors for eotaxin (CCR3) and IL-5, IL-3, and granulocyte macrophage colony-stimulating factor are studied in humans, revealing that TPI ASM8 attenuates the allergen-induced increase in target gene mRNA and airway responses in subjects with mild asthma [39].

\section{ROLE IN COPD}

COPD is characterized by a chronic inflammatory response of the airways and lungs to noxious particles and gases, mostly cigarette smoke. Pathologically changes characteristic of COPD include airway wall thickening, peribronchial fibrosis, peribronchial lymphoid follicles and destruction of lung parenchyma. Although the mechanisms of these changes remain unclear, there is now growing evidence that the recruitment of inflammatory cells in response to cigarette smoke is largely regulated by chemokines as ligands for chemokine receptors, e.g. CCR5 and CCR6 (Fig. 3). In patients with COPD, several CC-chemokines like MIP-1 $\alpha$, MIP-3 $\alpha$ and RANTES are upregulated [40-42], suggesting the contribution of their respective receptors in the pathogenesis of the disease. Such results have also been demonstrated in chronic cigarette smoke-exposure model [43, 44]. There was a negative correlation between FEV(1) percentage of predicted, FEV(1)/FVC ratio, and the levels of chemokines analyzed [40].

In the airway infiltration of dendritic cells (DCs), CCR6 at mRNA and protein levels was highly expressed in COPD patients. The interaction between CCL20 (MIP-3 $\alpha$, the ligand for CCR6) and CCR6 provides a possible mechanism for accumulation of DCs in the lungs in COPD [42]. Major chemokine elements are produced by CD1a-positive DCs, of which levels of ligands for CCR5 and CXCR3 are correlated with disease severity. They potentially mediate CD8-positive T-cell infiltration during COPD progression, and CD1apositive mucosal-associated DCs may sustain CD8-positive T-cell recruitment and/or retention [41]. Those contributions of CCR5 and CCR6 have been further confirmed by an attenuated accumulation of inflammatory cells like macrophages, dendritic cells, neutrophils and CD8-positive Tlymphocytes upon cigarette smoke-exposure in gene knockout mice $[43,44]$. Moreover, mice deficient for CCR5 or CCR6 were found to be partially protected from the development of pulmonary emphysema [43, 44], However, cigarette smoke-induced airway wall remodeling still occurred, suggesting that the mechanisms of the airway inflammation may different from those for airway remodeling. Eotaxin and CCR3 were also found to be up-regulated and involved in the recruitment of eosinophils and CD4+ lymphocytes into the airways which occur during acute exacerbations of chronic bronchitis [45]. These evidences suggest that chemokine receptors are potential therapeutic targets to reduce the chronic inflammation and parenchymal destruction in COPD.

\section{ROLE IN ALI/ARDS}

ALI and ARDS not only are critical cases of respiratory medicine, but also complicate many disease states and are central components of the systemic diseases, such as shock, trauma, severe infection, acute pancreatitis [46]. The uniform pathologic features of ARDS may involve sequestration of activated inflammatory cells and microvascular injury within the lung. A linkage between the cytokine (e.g., TNF- $\alpha$ ) and chemokine systems in the genesis of these syndromes may be postulated [47]. The deletion of CCR1 receptor, receptor for MIP- $1 \alpha$ and RANTES was noticed to protect from pulmonary inflammation secondary to acute pancreatitis, associated with decreased levels of TNF- $\alpha$ [47]. It indicates that the activation of the CCR1 receptor is an early event in the systemic inflammatory response. Administration of CCR1 antagonist, BX471, significantly protected mice against lung injury associated with cerulein-induced pancreatitis by attenuating myeloperoxidase activity, an indicator of neutrophil recruitment, and lung morphological changes in histological sections [48].

These results suggest that $\mathrm{CC}$ chemokine receptors may be involved in neutrophil trafficking in humans [49-51]. However, the main neutrophil chemokine receptors are CXCR1 and CXCR2 in human [1], indicating that MIP-1 $\alpha$ and CCR1 may be involved in rodents, while IL-8 and CXCR-1 in humans [47]. Data from transgenic mice suggested that the MCP-1/CCR2 signalling pathway may be involved in the protection against hyperoxia-induced ALI by suppressing production of inducible nitric oxide synthase and reactive oxygen species by activated alveolar macrophages [52].

\section{AIRWAY REMODELING}

Airway remodeling in asthma generally includes the increase of epithelial players, basement membrane thickening, extracellular matrix deposition, and goblet cell and ASMC number and size [53]. CCRs are proposed to play essential role in the pathogenesis of airway inflammation in asthma. For example, the CCR1/CCL3 axis was proposed to be associated with the development of the remodeling in a murine model of chronic fungal-induced allergic airway disease [54]. Significantly lower levels of Th2 cytokines were observed in the lungs of CCR1 deficient mice compared with their wild type counterparts, which correlated with significantly less fibrosis. This was confirmed by the therapeutic effect of CCR1 antagonist in a model of chronic fungal asthma [38]. Similar function of CCR8 was also noticed by altering the innate immune response [28].

CCR3 was found to play a role in the allergic airway inflammation and remodeling in the IL-13 gene-modified model of airway remodeling [55], evidenced by a clear reduction of IL-13-induced eosinophil recruitment into the lung lumen in the absence of eotaxin-2 or CCR3. It was correlated with attenuation in IL-13-induced mucus cell metaplasia and collagen deposition. In vitro studies demonstrated that CCR3 has a direct and selective profibrogenic effect on lung and bronchial fibroblasts [56], a novel mechanism by 
which eotaxin/CCR3 pathway may be involved in airway remodeling in asthma. In addition, the preventive effect of CCR3 antagonist on airway remodeling was noted in a chronic model of asthma [20]. Antagonizing CCR3 may be a new approach toward a promising asthma therapy.

\section{ROLE IN FIBROSIS}

Pulmonary fibrosis is characterized by the accumulation of fibroblasts, myofibroblasts, collagen, and other extracellular matrix proteins in the interstitial tissue of the lung, with subsequent scarring and destruction of the alveolar capillary interface. CCRs may play an important role in the pathogenesis of interstitial lung disease and be a potential tool for the disease treatment. MCP-1 was found to stimulate fibroblast collagen expression via specific receptors and endogenous up-regulation of TGF- $\beta$ expression [57]. CCR2(-/-) mice had less lung fibrosis in both the FITC and bleomycin pulmonary fibrosis models, accompanied by increased levels of GM-CSF and reduced levels of TNF- $\alpha$ [58]. CCR2 deficiency may improve the outcome of the disease by downregulating macrophage infiltration, macrophage-derived MMP-2 and MMP-9 production, fibrogenic cytokine expression and fibroblast responsiveness to TGF- $\beta[59,60]$.

Over-expression of CCR7 was found in idiopathic interstitial pneumonias and primary fibroblast lines, and systemic immunoneutralization of either CCR7 or its ligand significantly attenuated the pulmonary fibrosis in a mouse model of pulmonary fibrosis [61]. The exposure of CCL21 induced a significant migratory and proliferating response, which was inhibited by pertussis toxin or CCR7 antibodies [62]. CCR3 is considered to be involved in the development of lung fibrosis, evidenced by a significant protective function of blocking CCL11 and CCR3 interaction in mice [63].

\section{EXPERT COMMENTARY}

Chemokines act as regulatory molecules responsible for leukocyte maturation, trafficking, homing and inflammation in respiratory diseases. It is clear that chemokines and their receptors play the critical role in the initiation and development of respiratory diseases, possibly associated with the prognosis of patients, evidenced by the antagonism of the chemokine-CCR network. The inhibition of one single chemokine/chemokine receptor axis or knockout of the single gene has partial effects on the inhibition of these responses. The challenge for us is to discover and develop wider inhibition of CCRs and reach the optimal therapeutic or preventive efficacy with the least side effect. Thus, it is important to understand the potential role of CCRs in respiratory diseases and it may provide exciting new targets for therapeutic intervention.

\section{FIVE-YEAR VIEW}

It is important to prove the involvement of CCRs in the pathogenesis of lung diseases, but even more valuable to understand the correlation of CCR intracellular signal with lung diseases, duration, severity and prognosis. It is also important to clarify the significance of CCRs in the pathogenesis of lung diseases, either as a critical and unique player, an assistant or accelerator. There is a great need to discover and develop drugs interfering chemokine network or CCR inhibitor treating or preventing human diseases. It is reasonable for us to be positive on the future of this strategy, even though there is the possibility that inhibition of CCRs may lead to disruption of immune defense and immune surveillance, causing other drug-secondary severe infection or tumor. Thus, it is important to increase the understanding of the potential role of CCRs in human diseases and find appropriate approach to reach the balance and develop new drugs for therapeutic intervention.

\section{ACKNOWLEDGEMENT}

Authors thank BingRui Yang and YiChen Jia for their contributions on the preparation of figures. This work was supported by grants from the Shanghai Leading Academic Discipline Project (Project Number: B115).

\section{FINANCIAL \& COMPETING INTEREST DISCLO- SURE}

The authors have no relevant affiliations or financial involvement with any organization or entity with a financial interest in or financial conflict with the subject matter or materials discussed in the manuscript. This includes employment, consultancies, honoraria, stock ownership or options, expert testimony, grants or patents received or pending, or royalties.

\section{KEY ISSUES}

1. Chemokine receptors are not only expressed by circulating cells, but also by tissue resident cells, such as ASMC, to play an important role during episodes of tissue inflammation and injury, and in the pathogenesis of diseases.

2. There is strong evidence to confirm the role of chemokines and their receptors, including CCR3, CCR4, CCR8, and CCR1, in the development of allergic inflammation.

3. Recruitment of inflammatory cells in response to cigarette smoke is largely regulated by chemokines as ligands for chemokine receptors, e.g. CCR5 and CCR6.

4. CCR1 may be involved in the pathogenesis of ALI/ARDS via neutrophil trafficking, CCR2, CCR8 and CCR3 are considered as players in the development of lung fibrosis.

5. There is a clear need to develop CCR-specific inhibitors and figure out the balance between the effects and toxicities. It is also crucial to have a humanized in vivo system to screen the drug efficacy and binding, since it is common that the phenotypes of CCRs in animals differ from those of humans.

\section{REFERENCES}

[1] Viola A, Luster AD. Chemokines and their receptors: Drug targets in immunity and inflammation. Annu Rev Pharmacol Toxicol 2008; 48: 171-97.

[2] Baggiolini M. Chemokines and leukocyte traffic. Nature 1998; 392 565-8.

[3] Mariani M, Panina-Bordignon P. Analysis of homing receptor expression on infiltrating leukocytes in disease states. J Immunol Methods 2003; 273: 103-14.

[4] Doerschuk CM. Leukocyte trafficking in alveoli and airway passages. Respir Res 2000; 1: 136-40.

[5] Palmqvist C, Wardlaw AJ, Bradding P. Chemokines and their receptors as potential targets for the treatment of asthma. $\mathrm{Br} \mathrm{J}$ Pharmacol 2007; 151: 725-36. 
[6] Garcia G, Godot V, Humbert M. New chemokine targets for asthma therapy. Curr Allergy Asthma Rep 2005; 5: 155-60.

[7] Morgan AJ, Symon FA, Berry MA, et al. IL-4-expressing bronchoalveolar $\mathrm{T}$ cells from asthmatic and healthy subjects preferentially express CCR 3 and CCR 4. J. Allergy Clin Immunol 2005; 116: 594-600.

[8] Panina-Bordignon P, D'Ambrosio D. Chemokines and their receptors in asthma and chronic obstructive pulmonary disease. Curr Opin Pulm Med 2003; 9: 104-10.

[9] Joubert P, Lajoie-Kadoch S, Labonté I, et al. CCR3 expression and function in asthmatic airway smooth muscle cells. J Immunol 2005; 175: 2702-8.

[10] Pease JE. Asthma, allergy and chemokines. Curr Drug Targets 2006; 7: 3-12.

[11] Humbles AA, Lu B, Friend DS, et al. The murine CCR3 receptor regulates both the role of eosinophils and mast cells in allergeninduced airway inflammation and hyperresponsiveness. Proc Natl Acad Sci USA 2002; 99: 1479-84.

[12] Ma W, Bryce PJ, Humbles AA, et al. CCR3 is essential for skin eosinophilia and airway hyperresponsiveness in a murine model of allergic skin inflammation. J Clin Invest 2002; 109: 621-8.

[13] Pope SM, Zimmermann N, Stringer KF, et al. The eotaxin chemokines and CCR3 are fundamental regulators of allergeninduced pulmonary eosinophilia. J Immunol 2005; 175: 5341-50.

[14] Fryer AD, Stein LH, Nie Z, et al. Neuronal eotaxin and the effects of CCR3 antagonist on airway hyperreactivity and M2 receptor dysfunction. J Clin Invest 2006; 116: 228-36.

[15] Farahi N, Cowburn AS, Upton PD, et al. Eotaxin-1/CC chemokine ligand 11: a novel eosinophil survival factor secreted by human pulmonary artery endothelial cells. J Immunol 2007; 179: 1264-73.

[16] Moon KA, Kim SY, Kim TB, et al. Allergen-induced CD11b+ CD11c(int) CCR3+ macrophages in the lung promote eosinophilic airway inflammation in a mouse asthma model. Int Immunol 2007; 19: 1371-81.

[17] Tliba O, Amrani Y, Panettieri RA. Is airway smooth muscle the "missing link" modulating airway inflammation in asthma? Chest 2008;133: 236-42.

[18] Shen HH, Xu F, Zhang GS, et al. CCR3 monoclonal antibody inhibits airway eosinophilic inflammation and mucus overproduction in a mouse model of asthma. Acta Pharmacol Sin 2006; 27: 1594-9.

[19] Das AM, Vaddi KG, Solomon KA, et al. Selective inhibition of eosinophil influx into the lung by small molecule CC chemokine receptor 3 antagonists in mouse models of allergic inflammation. $\mathbf{J}$ Pharmacol Exp Ther 2006; 318: 411-7.

[20] Wegmann M, Göggel R, Sel S, et al. Effects of a low-molecularweight CCR-3 antagonist on chronic experimental asthma. Am J Respir Cell Mol Biol 2007; 36: 61-7.

[21] Fortin M, Ferrari N, Higgins ME, et al. Effects of antisense oligodeoxynucleotides targeting CCR3 on the airway response to antigen in rats. Oligonucleotides 2006; 16: 203-12.

[22] Panina-Bordignon P, Papi A, Mariani M, et al. The C-C chemokine receptors CCR4 and CCR8 identify airway $\mathrm{T}$ cells of allergenchallenged atopic asthmatics. J Clin Invest 2001; 107: 1357-64.

[23] Bonecchi R, Sozzani S, Stine JT, et al. Divergent effects of interleukin-4 and interferon- $\gamma$ on macrophage-derived chemokine Production: An amplification circuit of polarized T helper 2 responses. Blood 1998; 92: 2668-71.

[24] Meyer EH, Wurbel MA, Staton TL, et al. iNKT cells require CCR4 to localize to the airways and to induce airway hyperreactivity. J Immunol 2007; 179: 4661-71.

[25] Andrew DP, Ruffing N, Kim CH, et al. C-C chemokine receptor 4 expression defines a major subset of circulating nonintestinal memory T cells of both Th1 and Th2 potential. J Immunol 2001; 166: 103-11.

[26] Carpenter KJ, Hogaboam CM. Immunosuppressive effects of CCL17 on pulmonary antifungal responses during pulmonary invasive aspergillosis. Infect Immun 2005; 73: 7198-207.

[27] Gonzalo JA, Qiu Y, Lora JM, et al. Coordinated involvement of mast cells and $\mathrm{T}$ cells in allergic mucosal inflammation: critical role of the CC chemokine ligand 1:CCR8 axis. J Immunol 2007; 179: 1740-50.

[28] Buckland KF, O'connor EC, Coleman EM, et al. Remission of chronic fungal asthma in the absence of CCR8. J Allergy Clin Immunol 2007; 119: 997-1004.
[29] Mellado M, Martín de Ana A, Gómez L, et al. Chemokine receptor 2 blockade prevents asthma in a cynomolgus monkey model. J Pharmacol Exp Ther 2008; 324: 769-75.

[30] Yamashita N, Tashimo H, Matsuo Y, et al. Role of CCL21 and CCL19 in allergic inflammation in the ovalbumin-specific murine asthmatic model. J Allergy Clin Immunol 2006; 117: 1040-6.

[31] Hintzen G, Ohl L, del Rio ML, et al. Induction of tolerance to innocuous inhaled antigen relies on a CCR7-dependent dendritic cell-mediated antigen transport to the bronchial lymph node. J Immunol 2006; 177: 7346-54.

[32] Grinnan D, Sung SS, Dougherty JA, et al. Enhanced allergeninduced airway inflammation in paucity of lymph node T cell (plt) mutant mice. J Allergy Clin Immunol 2006; 118: 1234-41.

[33] Kaur D, Saunders R, Berger P, et al. Airway smooth muscle and mast cell-derived CC chemokine ligand 19 mediate airway smooth muscle migration in asthma. Am J Respir Crit Care Med 2006; 174: $1179-88$.

[34] Sen Y, Yongyi B, Yuling H, et al. V alpha 24-invariant NKT cells from patients with allergic asthma express CCR9 at high frequency and induce Th2 bias of CD3+ T cells upon CD226 engagement. J Immunol 2005; 175: 4914-26.

[35] John AE, Gerard CJ, Schaller M, et al. Respiratory syncytial virusinduced exaggeration of allergic airway disease is dependent upon CCR1-associated immune responses. Eur J Immunol 2005; 35: 108-16.

[36] Schaller MA, Kallal LE, Lukacs NW. A key role for CC chemokine receptor 1 in T-cell-mediated respiratory inflammation. Am J Pathol 2008; 172: 386-94.

[37] Joubert P, Lajoie-Kadoch S, Welman M, et al. Expression and regulation of CCR1 by airway smooth muscle cells in asthma. J Immunol 2008; 180: 1268-75.

[38] Carpenter KJ, Ewing JL, Schuh JM, et al. Therapeutic targeting of CCR1 attenuates established chronic fungal asthma in mice. Br J Pharmacol 2005; 145: 1160-72.

[39] Gauvreau GM, Boulet LP, Cockcroft DW, et al. Antisense therapy against CCR3 and the common beta chain attenuates allergeninduced eosinophilic responses. Am J Respir Crit Care Med 2008; 177: 952-8.

[40] Costa C, Rufino R, Traves SL, et al. CXCR3 and CCR5 chemokines in induced sputum from patients with COPD. Chest 2008; 133: 26-33.

[41] Freeman CM, Curtis JL, Chensue SW. CC chemokine receptor 5 and CXC chemokine receptor 6 expression by lung CD8+ cells correlates with chronic obstructive pulmonary disease severity. Am J Pathol 2007; 171: 767-76.

[42] Demedts IK, Bracke KR, Van Pottelberge G, et al. Accumulation of dendritic cells and increased CCL20 levels in the airways of patients with chronic obstructive pulmonary disease. Am J Respir Crit Care Med 2007; 175: 998-1005.

[43] Bracke KR, D'hulst AI, Maes T, et al. Cigarette smoke-induced pulmonary inflammation and emphysema are attenuated in CCR6deficient mice. J Immunol 2006; 177: 4350-9.

[44] Bracke KR, D'hulst AI, Maes T, et al. Cigarette smoke-induced pulmonary inflammation, but not airway remodelling, is attenuated in chemokine receptor 5-deficient mice. Clin Exp Allergy 2007; 37: 1467-79.

[45] Bocchino V, Bertorelli G, Bertrand CP, et al. Eotaxin and CCR3 are up-regulated in exacerbations of chronic bronchitis. Allergy 2002; $57: 17-22$

[46] Bone RC. Immunologic dissonance. Ann Intern Med 1996; 125 : 680-7.

[47] Gerard C, Frossard JL, Bhatia M, et al. Targeted disruption of the $\beta$ -chemokine receptor CCR1 protects against pancreatitis-associated lung injury. J Clin Invest 1997; 100: 2022-7.

[48] He M, Horuk R, Bhatia M. Treatment with BX471, a nonpeptide CCR1 antagonist, protects mice against acute pancreatitisassociated lung injury by modulating neutrophil recruitment. Pancreas 2007; 34: 233-41.

[49] Bonecchi R, Polentarutti N, Luini W, et al. Up-regulation of CCR1 and CCR3 and induction of chemotaxis to CC chemokines by IFN$\gamma$ in human neutrophils. J Immunol 1999; 162: 474-9.

[50] Cheng SS, Lai JJ, Lukacs NW, et al. Granulocyte-macrophage colony stimulating factor up-regulates CCR1 in human neutrophils. J Immunol 2001; 166: 1178-84.

[51] Lee SC, Brummet ME, Shahabuddin S, et al. Cutaneous injection of human subjects with macrophage inflammatory protein-1 $\alpha$ in- 
duces significant recruitment of neutrophils and monocytes. J Immunol 2000; 164: 3392-401.

[52] Okuma T, Terasaki Y, Sakashita N, et al. MCP-1/CCR2 signalling pathway regulates hyperoxia-induced acute lung injury via nitric oxide production. Int J Exp Pathol 2006; 87: 475-83.

[53] Boxall C, Holgate ST, Davies DE. The contribution of transforming growth factor- $\beta$ and epidermal growth factor signalling to airway remodelling in chronic asthma. Eur Respir J 2006; 27: 208-29.

[54] Blease K, Mehrad B, Standiford TJ, et al. Airway remodeling is absent in CCR1-/- mice during chronic fungal allergic airway disease. J Immunol 2000; 165: 1564-72.

[55] Fulkerson PC, Fischetti CA, Rothenberg ME. Eosinophils and CCR3 regulate interleukin-13 transgene-induced pulmonary remodeling. Am J Pathol 2006; 169: 2117-26.

[56] Puxeddu I, Bader R, Piliponsky AM, et al. The CC chemokine eotaxin/CCL11 has a selective profibrogenic effect on human lung fibroblasts. J Allergy Clin Immunol 2006; 117: 103-10.

[57] Gharaee-Kermani M, Denholm EM, Phan SH. Costimulation of fibroblast collagen and transforming growth factor betal gene expression by monocyte chemoattractant protein-1 via specific receptors. J Biol Chem 1996; 271: 17779-84.
[58] Moore BB, Paine R 3rd, Christensen PJ, et al. Protection from pulmonary fibrosis in the absence of CCR2 signaling. J Immunol 2001; 167: 4368-77.

[59] Okuma T, Terasaki Y, Kaikita K, et al. C-C chemokine receptor 2 (CCR2) deficiency improves bleomycin-induced pulmonary fibrosis by attenuation of both macrophage infiltration and production of macrophage-derived matrix metalloproteinases. J Pathol 2004; 204 : 594-604.

[60] Gharaee-Kermani M, McCullumsmith RE, Charo IF, et al. CCchemokine receptor 2 required for bleomycin-induced pulmonary fibrosis. Cytokine 2003; 24: 266-76.

[61] Pierce EM, Carpenter K, Jakubzick C, et al. Therapeutic targeting of CC ligand 21 or $\mathrm{CC}$ chemokine receptor 7 abrogates pulmonary fibrosis induced by the adoptive transfer of human pulmonary fibroblasts to immunodeficient mice. Am J Pathol 2007; 170: 115264.

[62] Pierce EM, Carpenter K, Jakubzick C, et al. Idiopathic pulmonary fibrosis fibroblasts migrate and proliferate to $\mathrm{CC}$ chemokine ligand 21. Eur Respir J 2007; 29: 1082-93.

[63] Huaux F, Gharaee-Kermani M, Liu T, et al. Role of Eotaxin-1 (CCL11) and CC chemokine receptor 3 (CCR3) in bleomycininduced lung injury and fibrosis. Am J Pathol 2005; 167: 1485-96.

Received: November 12, 2008

(C) Tong et al.; Licensee Bentham Open.

This is an open access article licensed under the terms of the Creative Commons Attribution Non-Commercial License (http://creativecommons.org/licenses/by-nc/3.0/) which permits unrestricted, non-commercial use, distribution and reproduction in any medium, provided the work is properly cited. 